\title{
Erratum: "Nanoindentation of bone: Comparison of specimens tested in liquid and embedded in polymethylmethacrylate" [J. Mater. Res. 19, 249 (2004)]
}

\author{
A.J. Bushby ${ }^{a)}$ \\ Department of Materials, Queen Mary, University of London, London E1 4NS, United Kingdom \\ V.L. Ferguson \\ Department of Materials, Queen Mary, University of London, London E1 4NS, United Kingdom; \\ and Department of Anatomy and Developmental Biology, University College London, \\ London WC1E 6BT, United Kingdom
}

\author{
A. Boyde \\ Department of Anatomy and Developmental Biology, University College London, \\ London WC1E 6BT, United Kingdom; and Dental Biophysics, Centre for Oral Growth and \\ Development, Queen Mary, University of London, Institute of Dentistry, London E1 2AD, \\ United Kingdom
}

(Received 25 February 2004; accepted 25 February 2004)

After this article appeared in the January 2004 issue of Journal of Materials Research, the authors noticed that Fig. 6(b) was incorrect. The correct figure is shown below.

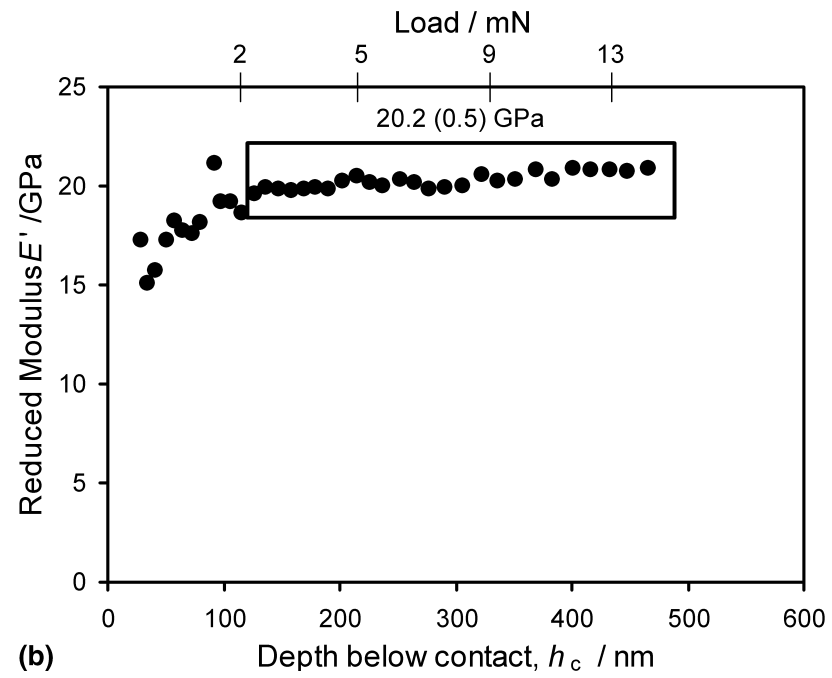

FIG. 6. $E^{\prime}$ versus $h_{\mathrm{c}}$ for the (a) $R=21-\mu \mathrm{m}$-radius indenter, wet (SCL) conditions, and (b) $R=5$ - $\mu \mathrm{m}$-radius indenter, embedded condition, illustration that the low stiffness surface layer is eliminated in PMMA. Mean $E^{\prime}(\mathrm{SD})$ presented for each case is calculated from 25 data points shown in the boxed regions. The indentation loads are plotted along the top axis as a guide only because the loads are not linearly proportional to $h_{\mathrm{c}}$. Solid line in (a) is the finite element model of Fig. 7 plotted as $E^{\prime}$ versus $h_{\mathrm{c}}$ for comparison to show that the experimental data can be interpreted using a thin-film model.

\footnotetext{
a) Address all correspondence to this author.

e-mail: a.j.bushby@qmul.ac.uk

DOI: 10.1557/JMR.2004.0211
} 